\title{
"WAZZ Up Doc?" Cost Efficient, User Friendly, Patient Trust Level Based on Convenience and the Use of Hypnotherapy Mobile Apps Method
}

\author{
Imelda Imelda ${ }^{1}$, Dewi Puspaningtyas Faeni ${ }^{2}$, Ratih Puspaningtias Faeni ${ }^{3}$, \\ Rinny Meidiyustiani ${ }^{4}$, Retno Fuji Oktaviani ${ }^{5 *}$ \\ ${ }^{1}$ Faculty of Informatics Technology, Universitas Budi Luhur, Jakarta 12260, Indonesia \\ ${ }^{2,3,4,5}$ Faculty of Economics and Business, Universitas Budi Luhur, Jakarta 12260, Indonesia
}

\begin{abstract}
A B S T RAC T
Counseling method that uses a breakthrough unconscious forces, called hypnotherapy, is very effective in dealing with psychosomatic history. The reason patients' reluctance to visit specialist counseling and therapy; such a negative stigma, negative social perception, shame somebody to public judgment about mental illness, heavy traffic conditions and geographical distance between patient and psychologist, as well as the affordability of the financial condition of the patient. Effective solutions related to the matter is android based application development "WAZZ Up, Doc?" in the era of Society 5.0. These applications in particular have a very high accessibility and user friendly, convenient for $24 / 7$ accessibility and availability. The contribution of this research was to determine the effect of efficiency in transportation, user friendly, increasing the patient's level of confidence in the psychologist/psychiatrist and high accessibility comfort $24 / 7$ on the use of mobile applications hypnotherapy "WAZZ Up, Doc?". The method used is SEM-PLS with 3600 respondents.
\end{abstract}

ARTICLE INFO

Article History:

Received : 12-08-2019

Revised : 29-09-2019

Accepted : 10-10-2019

Published : 28-10-2019

\section{Keywords:}

User friendly,

Cost efficiency,

Patient trust levels,

Convenience,

Hypnotherapy,

Mobile apps,

$24 / 7$.

*Corresponding Author E-mail:

retno.fujioktaviani@budiluhur.ac.id

Copyright $@ 2019$ Authors. This is an open access article distributed under the Creative Commons Attribution License, which permits unrestricted use, distribution, and reproduction in any medium, provided the original work is properly cited.

\section{INTRODUCTION}

Psychosocial Indonesian society has a difficulty level that is so full of complexity. Problems that occur typically include: the difficulty of finding a job, patchy economic problems, personal problems are complicated by the difficulties currently survive in certain conditions. These difficulties are encouraging people to vent. Psychologists provide a professional service that handles counseling (Elahi et al., 2013).

These conditions encourage high psychosocial counseling needs in a professional and 
confidential, but the main obstacle is the psychological obstacles, economically and geographically to the availability of counseling. First, there is the negative stigma that someone counseling by visiting a psychologist or psychiatrist has been labeled as someone who is mentally ill. Second, the economic constraints that a visit to a psychologist / psychiatrist is still very expensive. Third, remote geographical constraints and bottlenecks greatly inhibited to achieve the goals of counseling.

Hypno healing or therapeutic hypnosis is a new breakthrough in technology and genial. Healing hypno make it easier for someone to find effective solutions and providing more adequate counseling services (Wang, 2016). This study provides the power of the subconscious and provide psychological solutions so that people in need can access the android-based technology applications real-time free from the constraints of sociological, economic, and geographic. This research resulted in the analysis of the effect of someone who has been using mobile apps "WAZZ Up, Doc?". "WAZZ Up, Doc?" mobile apps are accessible to everyone who provides $24 / 7$ support services such as 911 . The contribution of this study was to determine the effect of transportation cost efficiency, user friendliness, increased levels of patient trust in the psychologist/psychiatrist and high accessibility $24 / 7$ convenience to use hypnotherapy mobile apps "WAZZ Up, Doc?". The method used in this study is the SEM-PLS. The formula used is Slovin as a determinant of respondents was 3,600. The final result of the calculation Slovin respondent is 97 people.

\section{MOBILE APPS HYPNOTERAPY METHOD}

Technological breakthrough in this research that shown in Figure 1 is to see the effect caused by the change of technology from conventional hypnotherapy to Mobile Apps. The conventional Hypnotherapy has the following phases: pre-talk, problem identification, relaxation, thought patterns of rest, physical rest, give suggestions to the subconscious and enlightenment (provide barrier in the form of Post suggestion). Hypnotherapy mobile apps "WAZZUp, Doc?" is the identification of users by registering as a user and create a password, the identification problem by selecting the appropriate menu the patient's condition, start by turning on audio hypnosis therapy so as to balance a person's soul.

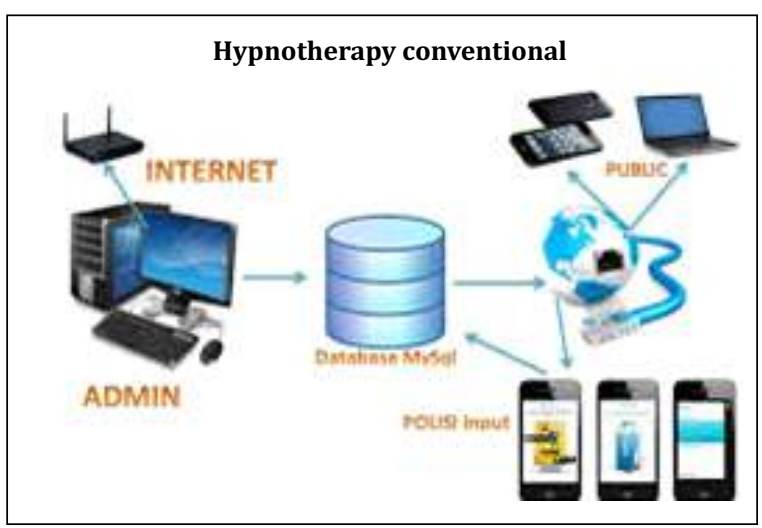

Figure 1. Technology Infrastructure from Manual to Mobile Apps "WAZZ Up, Doc?"

Hypnosis method using mobile apps is very helpful because someone immediately get help as needed today without face-to-face with a psychologist. This solution fits perfectly with the cultural conditions of Indonesia, which is the eastern culture. Eastern cultures tend not allowed to look weak of others. According to the Eastern culture, it is not good to open shame in the face of others. This is because culture is not a culture of counseling Indonesia. "WAZZ Up, Doc?" provide solutions that can serve the souls of all people of Indonesia and is always ready to provide assistance 24 hours in 7 days, such as service 911. Mobile apps provide convenience, systematic and flexible. Ease because transport costs are very cheap. More systematic because it directly refers to the patient's needs. Flexible because of the existence of these mobile apps really help provide therapists solution for those who need anytime and anywhere without interruption 24 hours a week. Mobile apps serve as a Service Hot Line which is accessible 24/7 and Call Center "WAZZ Up, Doc?" Online counseling services.

The behavior of a person like an iceberg, which looks like a small hill above the water, but it contains a very large ice below the water surface is shown in Figure 2. The small ice hill that appears above the water level are aware of a person's 
behavior, otherwise known as the conscious mind, the content of the ice below sea level is the contents of the subconscious or more popular with the term unconscious. The analogy of an iceberg that is shown in Figure 2 gives the conclusion, that the unconscious that appeared in the person's behavior is shaped by a person's subconscious. Figure 2 on the right side shows a person's consciousness is greatly controlled by the subconscious that stores all the data bases of the empirical experience of a person. Subconscious move triggered based on past learning. Empirical experience and past learning into one's values and shape a person's behavior. Figure 2 on the left side shows how a person reacts when threatened, the person's behavior when threatened. There are two kinds of reactions, namely, the sympathetic and parasympathetic reaction to inhibition of the normal defense mechanisms are under threat. Sympathetic when someone is threatened, there arose a disproportionate reaction. Sympathetic reaction in question can be categorized into two (2) types of reactions, fly and fight. Flights are in the form of depression, psychological conditions repressive or one of them is to avoid, stress to the protracted problem without resolution. Fight react aggressively to resolve the problem. For example: a tantrum in a public place, attacking anyone in the vicinity or even do things that are artificial and counterproductive. Both types of sympathetic reaction will be very alarming if left in the long term, psychotic and depressive symptoms will appear as an indicator of a person in a mental imbalance. Meanwhile, the parasympathetic action is contrary actions, one's mental maturity and balance in the face of problems can be faced with calm and even stay focused on what it does. This is an indicator of parasympathetic actions clinical people who have mental health balanced (Fishburn, 1967). Psychosocial Indonesian society is considered full complexity. Psychosocial distress caused numerous problems including: difficulty finding jobs, the economic problems that ensnare, personal problems complicated by the difficulties currently survive in certain conditions Schreiber (Elliott, 2016). The difficulties that encourages people to seek counseling with professional psychological assistance service (Elahi etal., 2013).

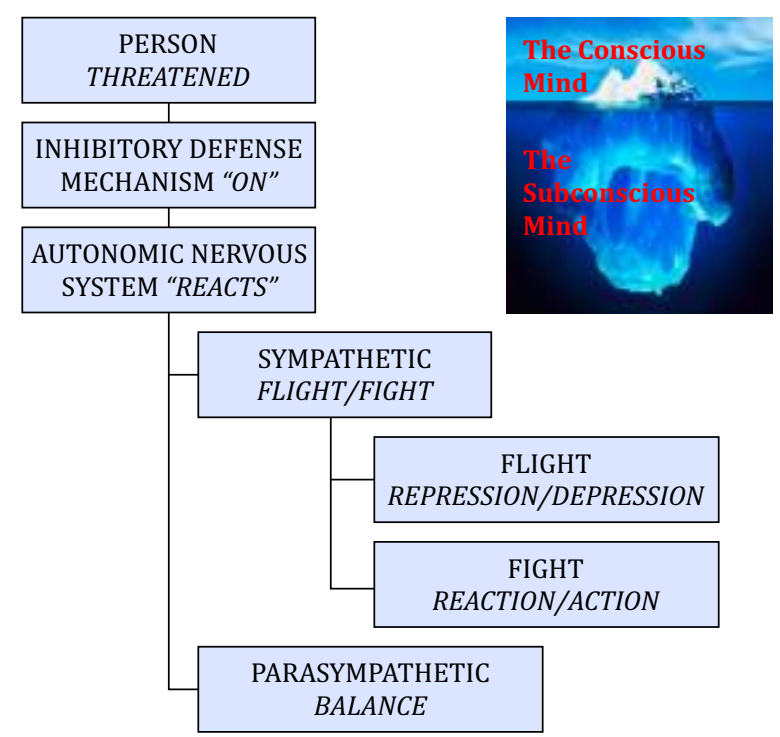

Figure 2. Behavior Formed by the Subconscious (Schreiber, 2016)

\section{RESULT METHODS}

\section{Data Analysis Techniques}

Analysis of the data used this research is the approach Partial Least Square (PLS) PLS is a model for Structural Equation Modeling (SEM) based component or a variant. According Ghozali (2011) [6], PLS is an alternative approach that shifts the approach to the covariance-based SEMbased variants. Whereas a more predictive PLS model covariance-based SEM generally test the causality/theory. PLS is a powerful analytical method (Ghozali. 2011), the sample should not be large because it is not based on many assumptions that are normally distributed.

The method used is SEM-PLS. Slovin formula used to determine the respondent. The object of this study is 3600 patients with hypnosis. The final result of the calculation formula Slovin is 97 people.

\section{RESULTS AND DISCUSSION}

\section{Evaluation of Outer Models}

Outside measurement models or models with reflective indicators were evaluated by means of convergent and discriminant validity and reliability of the composite indicator for the block of indicators (Faeni \& HypnoBeauty, 2016). 
The initial models of this research is as follows: user friendly ( $\mathrm{x} 1$ ) is measured by four reflective indicators, cost efficiency (x2) are measured by four reflective indicators, patient trust levels $(\mathrm{x} 3)$ is measured by four reflective indicators, convenience ( $\mathrm{y} 1)$ is measured by four reflective indicators and application usage (y2) is measured by six reflective indicators.

The indicators of User friendly are: (1) I think the "WAZZ Up, Doc?" Hypnotherapy Mobile apps is very easy to use. (2) The use of the "WAZZ Up, Doc?" Hypnotherapy Mobile apps is so easy that I am Able to do it myself without the help of others. (3) The "WAZZ Up, Doc?" Hypnotherapy Mobile apps is very easy to operate and so I do not feel any Difficulties. (4) The operation of the "WAZZ Up, Doc?" Hypnotherapy Mobile apps is very light and easy so I like to use it.

The indicators of Cost efficiency are: (1) The operation of "WAZZ Up, Doc?" Hypnotherapy Mobile apps is very solutive and inexpensive, (2) The "WAZZ Up, Doc?" Hypnotherapy Mobile apps is very efficient to use in addition to being easy to pay very cheaply, (3) I was greatly helped by the "WAZZ Up, Doc?" Hypnotherapy Mobile apps because my time was more flexible, (4) I know the use of "WAZZ Up, Doc?" Hypnotherapy Mobile apps more efficiently (Ghozali. 2011).

The indicators of patient trust levels are: (1) "WAZZ Up, Doc?" Hypnotherapy Mobile apps can improve performance (2) "WAZZ Up, Doc?" Hypnotherapy Mobile apps can be increase productivity levels (3) "WAZZ Up, Doc?" Hypnotherapy Mobile apps can improve performance effectiveness (4) "WAZZ Up, Doc?" Hypnotherapy Mobile apps is able to treat my inner illness.

The indicators of Convenience are: (1) I feel better after hear the audio recording in this "WAZZ Up, Doc?" Hypnotherapy Mobile apps, (2) I am more productive after using this "WAZZ Up, Doc?" Hypnotherapy Mobile apps, (3) I feel freer after using this "WAZZ Up, Doc?" Hypnotherapy Mobile apps, (4) I can do things more focused than before productive after using this "WAZZ Up, Doc?"
Hypnotherapy Mobile apps.

The indicators of Use of "WAZZ Up, Doc?" Hypnotherapy Mobile apps are: (1) I am interested in using "WAZZ Up, Doc?" Hypnotherapy Mobile apps because the features offered are complete and interesting. (2) The "WAZZ Up, Doc?" Hypnotherapy Mobile apps makes it so easy that I'm comfortable using it. (3) I always try to use "WAZZ Up, Doc?" Hypnotherapy Mobile apps because it gives calm. (4) I always use "WAZZ Up, Doc?" Hypnotherapy Mobile apps when I am treated.

\section{Cross Loading}

Cross loading is construct correlation with measurement items that are larger than the size of the other constructs. The results show that the latent constructs predict the size of the block is better than the other block size (Cho and Sagynov, 2015).

In PLS, testing every relationship is done by using a simulation with bootstrapping method on samples. This test aims to minimize the problem of abnormalities of research data. Results of testing with bootstrapping method of PLS SEM analysis in Figure 3.

\section{Convergent Validity}

Individual reflective size is high if it correlates more than 0.70 with the measure of construct. However, for the initial stage of the study, the scale of measurement of loading values from 0:50 to 0.60 was considered sufficient (Cho and Sagynov, 2015).

Based on the measurement models in Figure 3, all indicators are analysis of research variables with a loading factor of greater than 0:50 so that it is declared significant or convergent validity meets requirements.

Meanwhile fo $r$ the calculation results can be viewed under the influence of direct, indirect and total in Table 1. Table 1 shows the results of the calculation stating PLS direct influence between variables. It is said there is a direct effect if the 


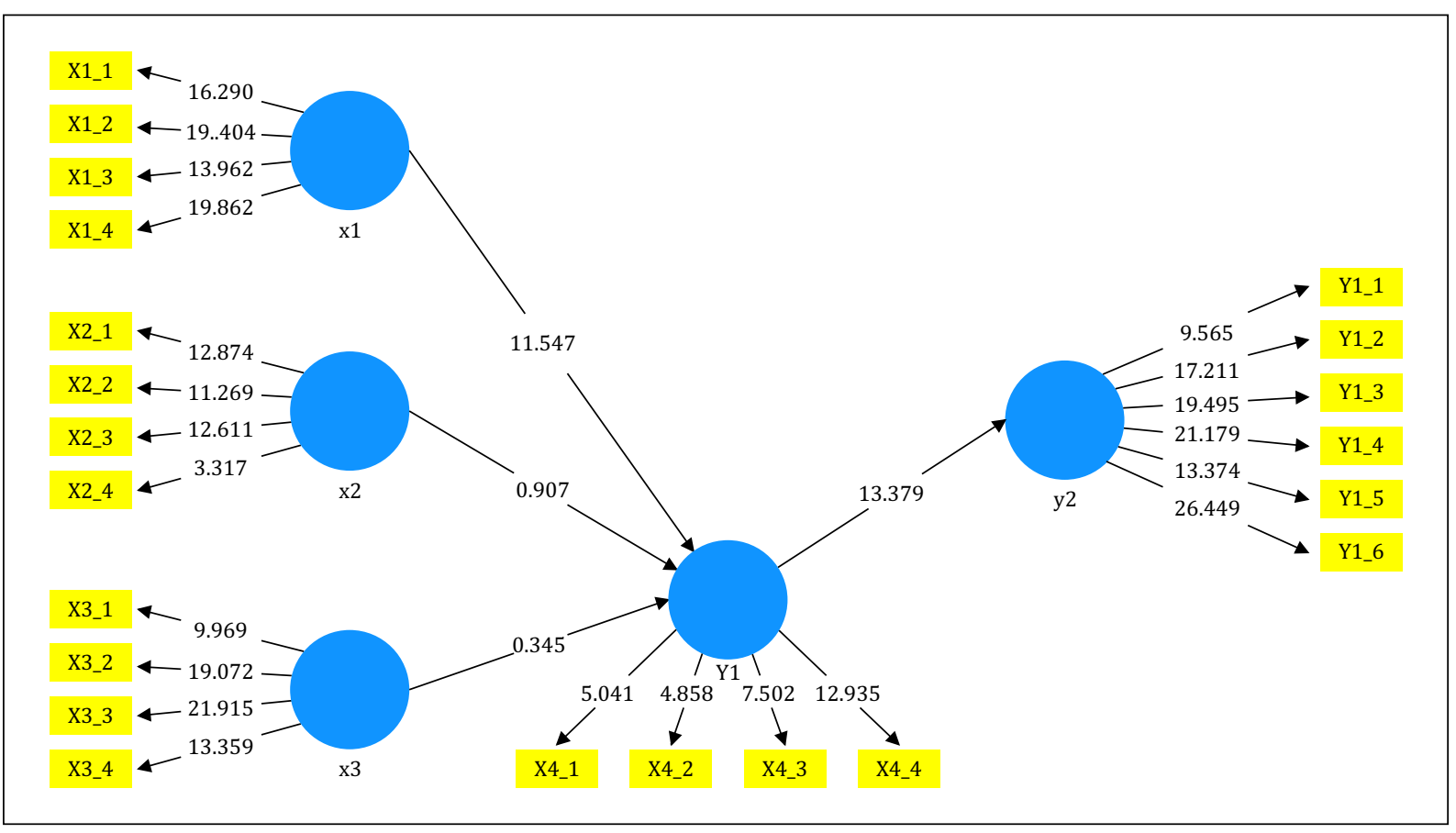

Figure 3. Convergent Validity

p-value $<0.05$ and in saying there is no direct effect if the $p$-value> 0.05. Significant variable to variable user-friendly convenience with 0.00 $\mathrm{p}$-value $<0.05$. Significant variable to variable user-friendly application usage with $0.00 \mathrm{p}$-value $<0.05$. Variables significant convenience to variable use of the application with p-value $0.000<0.05$. Variable cost-effective convenience not significant, with p-value $0.420>0.005$. Variable cost effective not significant to the usage of hypnotherapy mobile apps, with a p-value $0,436>0.005$. Variable patient trust level is not significant to the convenience, with p-value $0.7300>0.005$. Variable patient trust level is not significant to the usage of hypnotherapy mobile apps, with a p-value $0.732>0.005$.

Table 1. Total Effects

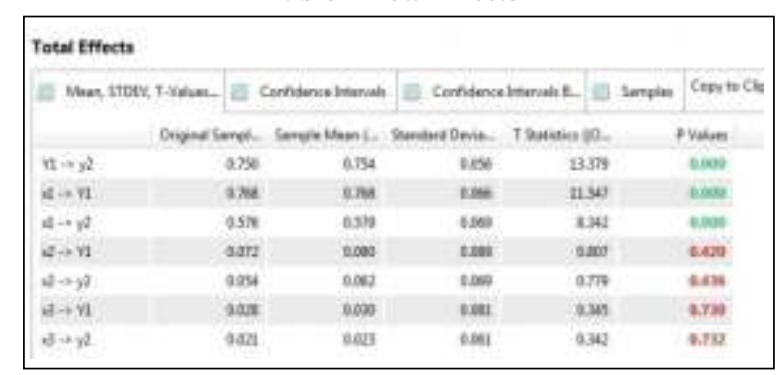

Source: Smart-PLS output

Table 2 shows the total indirect effect. User friendly has a significant influence indirectly to the use of the application. this can be seen from the P-values of $0.000<0.05$. Cost Efficiency has no significant influence indirectly to the use of the application. this can be seen from the P-values of $0.436>0.05$. Patient trust levels had no influence indirectly to the use of the application. this can be seen from the P-values of $0.736>0.05$.

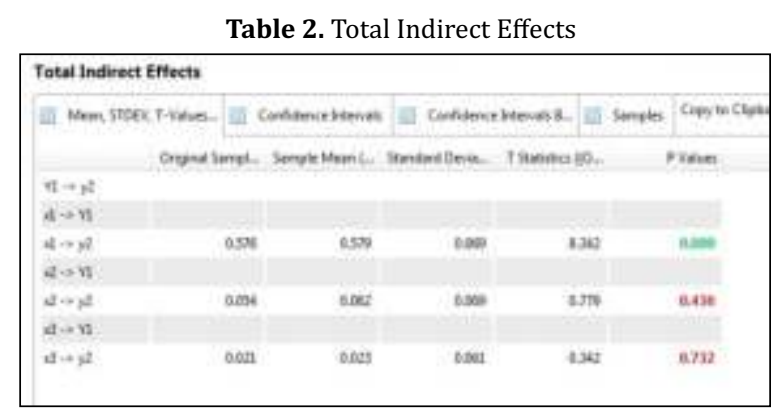

Source: Smart-PLS output

User friendly have significant influence indirectly to the use of mobile apps. User friendly is one of the factors that can affect one's attitude in using the application. One considers that the use of "WAZZ Up, Doc?" mobile apps is fun. It can be seen from the user ease of use, its complete and provide all the needs of the patient. Menu also be the best solution for the patient. With the ease of research, the patients feel comfortable and do not need face-to-face sessions. Ease of confidentiality occurs because patients could use an anonymous account and alter. All mental 
blocking will know the identity of patients to be small, so that anyone who assess the "WAZZ Up, Doc?" mobile apps will be felt comfort and confident. Distinct from the concept of conservative counseling.

\section{CONCLUSION}

The results of this research found that friendly user has a strong and significant effect on convenience. User friendly, has a strong, significant direct and indirect effects on mobile apps usage. Convenience has a strong and significant effect on mobile apps usage. The results of the research found that the cost efficiency Significantly did not influence the use of the mobile apps. Research results found the patient trust levels has not significantly indirect effect on the use of the hypnotherapy mobile apps "WAZZ Up, Doc?".

\section{REFERENCES}

Elahi, Z, Boostani, R \& Nasrabadi, Motie (2013). Estimation of Hypnosis Susceptinility Based On Electrencephalogram Signal Features, Scientica Iranica.

Wang., EH, (2016). AM Health Platform for Supporting Clinical Data Integration and Service Delivery: An Example From augmentative and Alternative Communication Intervention, dissertation, University of Pittsburgh.

Fishburn, PC (1967). Utilities with Incomplete Additive Product Set: Applications to Priorities and Assignments. American Society of Operations Research (ORSA), Baltimore, MD, USA Miller.

Schreiber, Elliott H. (2016). Use of Group Hypnosis to Improve College Students' Achievement, Sage Journals. Faeni, Dewi, Hypno Beauty (2016), Hypnosis for Women High Achievers, Qanita Publication.

Ghozali. I. (2011). Alternative Method of Structural Equation Modeling Metode Alternatif dengan Partial Least Square. Semarang: Universitas Diponegoro Publisher Agency.

Cho, YC., Sagynov, E. (2015). Exploring Factors That Affect Usefullness, ease of use, trust, and purchase intention in the online environment. International Journal of Management \& Information Systems, 19, 1 